\title{
The Scientific Targets: The Myocardium, the Vasculature and the Body's Response to Heart Failure
}

\author{
Rafael Vidal-Pérez¹, Ewa A Jankowska ${ }^{2,3}$ \\ 1. Cardiology Department, Heart Failure Unit, Hospital Clinico Universitario de Santiago de Compostela Spain \\ 2. Department of Heart Diseases, Wroclaw Medical University, Wroclaw, Poland \\ 3. Centre for Heart Diseases, University Hospital, Wrocław, Poland
}

\section{Corresponding author:}

Ewa A. Jankowska, Department of Heart Diseases, Wroclaw Medical University and Centre for Heart Diseases, University Hospital in Wroclaw, Poland.

Email: ewa.jankowska@am.wroc.pl

\section{Abstract}

Heart failure (HF) is a common but complex clinical syndrome associated with a reduced ability of a heart to pump and/or fill with blood. We now appreciate the more complex picture involving metabolic derangements, changes in foetal gene expression and abnormalities in the periphery as forming part of the HF syndrome. Therapeutic targets include the failing myocardium, the vasculature and peripheral mechanisms. The pathophysiology of HF is currently being intensively investigated, with the identification of new relevant mechanisms, some of them emerging as potential therapeutic targets.

Keywords: Heart failure; therapeutic targets

Citation: Jankowska E, Vidal-Pérez R. The Scientific Targets: the Myocardium, the Vasculature and the Body's Response to Heart Failure. International Cardiovascular Forum Journal. 2019;17:12-18. DOI: 10.17987/icfj.v17i0.593

\section{Introduction}

Heart failure (HF) is a common but complex clinical syndrome associated with a reduced ability of a heart to pump and/or fill with blood. HF may be defined pathophysiologically as a cardiac output inadequate to meet metabolic demands, or an adequate cardiac output secondary to excessive compensatory neurohormonal activation (generally manifested as increased left ventricular filling pressures).[1]

The theories explaining the origin of the HF syndrome are rapidly evolving, moving from the concept of hypervolaemia due to pump failure and consequent neurohormonal activation to a more complex picture involving metabolic derangements, changes in foetal gene expression and abnormalities in the periphery to name but a few. These other features of the HF syndrome are currently the object of much basic and clinical research (Figure 1).

\section{The failing myocardium as a scientific target}

When the heart starts to fail, a number of intrinsic mechanisms are invoked in order to increase cardiac output and thereby maintain adequate tissue perfusion. Initially, through the FrankStarling mechanism, preload can be increased by ventricular chamber dilation and volume expansion can thereby enhance cardiac output. This mechanism, however, becomes deleterious if maintained long-term.[2]

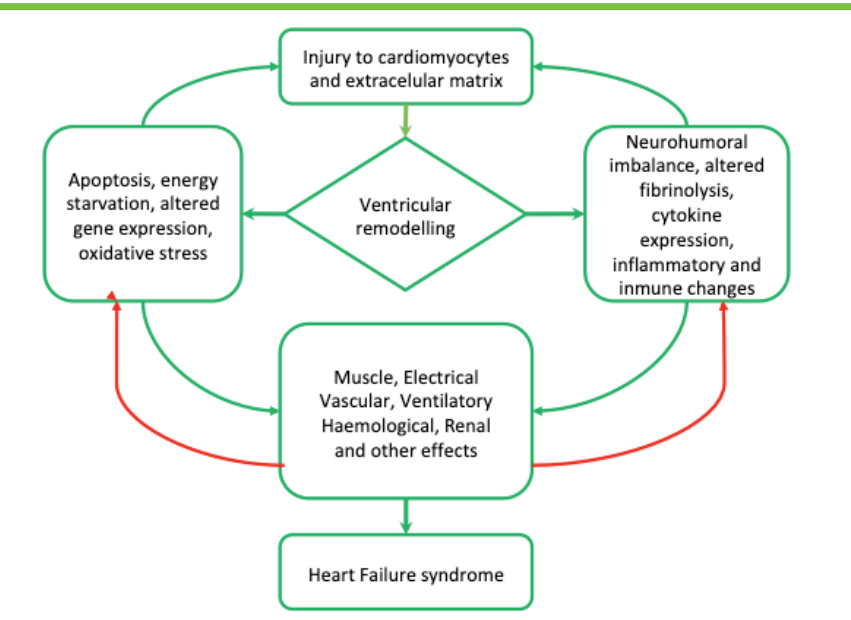

Figure 1. Pathophysiology of HF as a result of LV dysfunction. Damage to the cardiomyocytes and extracellular matrix leads to changes in the size, shape, and function of the left ventricle and heart more generally ("remodeling"). These changes, in turn, lead to electrical instability, systemic processes resulting in many effects on other organs and tissues, and further damage to the heart. These vicious cycles, along with intercurrent events, such as myocardial infarction or hypertensive crisis, are believed to cause progressive worsening of the syndrome of heart failure over time. 
There are two general models that attempt to unify and explain the origin of HF: heart damage (e.g. infarction, myocarditis) leading to stiffness, myocardial remodelling and ventricular dilatation (3), and heart hypertrophy (e.g. due to hypertension or other pressure or volume overload syndromes), where a myocardial hypertrophic response precedes later wall thinning and cavity remodelling.

When gathering evidence concerning the pathophysiology of $\mathrm{HF}$, an explicit separation between heart failure with preserved ejection fraction (HFpEF) and heart failure with reduced ejection fraction (HFrEF) seems arbitrary. The vast majority of patients with HF undergo the following steps in the progression of HF: 1) heart damage (of differing types in terms of aetiology, time and magnitude); 2) myocardial stiffness (with early changes in haemodynamics); 3) myocardial remodelling (local and/or global) with a preceding phase of hypertrophy in some cases - along with structural and functional pathologies - quantitative and qualitative changes on the histological and molecular level (including deranged cardiomyocytes, cardiofibroblasts, and other cell types); 4) further advanced changes in remodelling leading to wall thinning and ventricular/atrial dilatation with changes in geometry, dyssynchrony, and further metabolic and molecular changes in the myocardium.

In HFpEF (but also in the more recently defined HF with midrange (40-50\%) EF (HFmrEF), the primary functional abnormality is increased left ventricular stiffness due to a variety of processes including ischaemia, insulin resistance and fibrosis, which is associated with increased left ventricular diastolic pressure, a passive increase in left atrial and pulmonary venous pressure, that in turns causes signs and symptoms of pulmonary venous congestion. Pressure overload is associated with a parallel replication of myofibrils and thickening of individual cardiomyocytes as well as an activation of cardiofibroblasts with resulting changes in the extracellular matrix.

In HFrEF (but also to some extent in HFmrEF), there are marked changes in LV shape and geometry with the LV becoming more globular or spherical. Volume overload leads to a replication of sarcomeres in series and an elongation of myocytes, that proceed to a stretching of the mitral valve ring and consequent functional mitral regurgitation. This pathology significantly increases the load on the failing ventricle, and contributes to another 'vicious cycle', that can drive a cycle of deterioration and a progression of the manifestations of HF. An initial stress-induced increase in sarcomere length can maintain a near optimal overlap between myofilaments, but severe haemodynamic overload eventually yields to a depression of myocardial contractility.[3] Along with dilatation and an altered geometry of the cardiac chambers, another phenomenon is the presence of dyssynchrony, seen at the interventricle, interatrium and atrio-ventricular levels, the mechanical dyssynchrony is usually associated with electrical dyssynchrony, which usually appears first, particularly in a form of left bundle branch block on the ECG.

These structural and functional changes in HFpEF, HFmrEF and HFrEF translate into an adaptation (to a new haemodynamic status) of volumes and pressures within the atria and ventricles. In HFrEF, the major abnormality will be a reduced left ventricular ejection fraction (LVEF), associated with reduced stroke volume and increased LV end diastolic and LV end systolic volumes (LVEDV and LVESV).

The underlying causes of myocardial remodelling are the target of many established life-saving therapies for patients with HFrEF, such as renin angiotensin aldosterone system (RAAS) blockers (angiotensin converting enzyme inhibitors, angiotensin receptor type 2 blockers, also in combination with neprilysin inhibitors, and mineralocorticoid receptor antagonists), and beta-blockers. $[4,5]$ Therapeutic options to treat functional mitral regurgitation are currently also under investigation with different types of mitral devices [6] as well as cardiac resynchronization therapy (CRT). $[7,8]$ CRT has been designed primarily for counteracting different types of dyssynchrony, but importantly the consequences of effective resynchronization include improvements in left ventricular filling time and contractility, a decrease in mitral regurgitation and an increase in cardiac output.[7,8]

An important pathophysiological element of HFrEF in particular is impaired cardiac contractility seen at the molecular, cellular and structural levels.[9] Based on this paradigm, drugs increasing cardiac contractility (positive inotropes) were developed for the treatment of HF. The first generation of positive inotropes, including phosphodiesterase-inhibitors (milrinone) and catecholamine derivates (dobutamine, dopamine, norephinephrine), although used still in some circumstances, have several limitations due to serious side-effects. However, development continues in this area, and the novel positive inotropes (levosimendan, omecamtiv mecarbil) have a better safety profile with some potential regarding their efficacy.[10,11] Importantly, cardiac contractility modulation (CCM) represents a device-based intervention aiming to improve cardiac contractility and global myocardial function through the application of nonexcitatory electrical signals during the absolute refractory period, adjusted to and synchronized with the electrical action in the cardiac cycle.

The current understanding of molecular and metabolic mechanisms behind the aforementioned macroscopic changes remains limited. These changes may be localized after myocardial infarction, but more diffuse in patients with idiopathic dilated cardiomyopathy and/or myocarditis.[12] They comprise myocyte loss by necrosis and apoptosis, alterations in excitation-contraction coupling, alterations in composition and architecture of the extracellular matrix and deranged functioning of cardiac fibroblasts.[13] Apoptosis results from the induction of a genetic programme that leads to the degradation of nuclear DNA, which is induced due to angiotensin II, reactive oxygen species, nitric oxide, pro-inflammatory cytokines.[13] Changes in the extracellular matrix usually manifest with an increase in collagen content though the imbalance between degradation and synthesis (along with the changed activity of enzymes governing these processes).[14] Some drugs counteracting these processes are in development, e.g. anti-apoptotic agents or anti- matrix metalloproteinases.[15]

In myocardial tissue, within the set of expressed genes, certain genes representing sarcomere and mitochondrial components and other transcripts are abundantly expressed in the cardiomyocytes. The molecular profiling of the failing heart shows widespread changes in splicing of sarcomere genes 
with a transition from a-myosin to $\beta$-myosin expression, a titin isoform switch, a lower expression of key calcium channels including SERCA2a and RYR2, an up-regulation of natriuretic peptides, a switch from expression of enzymes involved in fatty acid oxidation to glycolysis as well as a switch from enzymes involved in oxidative reactions towards anaerobic processes with augmented production of reactive species.[16]

Of special importance for the origin of HF is the presence of specific profiles of microRNA (miRNA) expression. These miRNAs are short, highly conserved, anti-sense RNA strands that target mRNA in a complementary manner and reduce protein expression by inhibiting mRNA translation and by targeting their breakdown. Many of them are described in HF as a potential therapeutic target, i.e. some drugs could promote the degradation of some detrimental miRNAs or vice versa.[17]

There is emerging evidence indicating that epigenetic regulation may play an important role in the pathogenesis of HF, namely in the phenotypic response of a failing heart [18]. Epigenetics refers to the changes in the regulation of gene activity and its expression that are not related to the gene sequence. These epigenetic modifications affecting DNA methylation, ATP-dependent chromatin remodelling, histone modifications, and microRNA-related mechanisms are considered sufficient factors contributing to adverse cardiac remodelling and preceding overt cardiac dysfunction [19] (Figure 2). In the future epigenetic modifications of cardiomyocytes and/or cardiac fibroblasts could be a target for personalized management and more effective tools for the prevention of HF [20].

\section{The vasculature as a scientific target}

Abnormalities within vasculature occur in the course of HF, across the whole spectrum of LVEF. However, these pathophysiological changes in the vasculature are of special importance in patients with HFpEF.[22]

Microvascular dysfunction. Numerous pathophysiological mechanisms have been proposed for coronary microvascular dysfunction, as well as for peripheral microvascular dysfunction, contributing for example to the limitation of exertion-related perfusion of exercising muscles, and reduced global perfusion during episodes of decompensation. These changes include endothelial, smooth muscle, and sympathetic dysfunction, microvascular spasm, extramural rarefaction, and luminal obstruction, and all are potential targets for treatment interventions.[23]

Vascular remodelling and vasoconstriction. Patients with HF develop vascular remodelling that is related to hyperplasia of vascular smooth muscle cells, driven mainly by the activation of the RAAS. Additionally systemic as well as pulmonary vasoconstriction are fundamental features of HF progression, and are thought largely to be due to neurohormonal activation, inflammation and insulin resistance, with a resultant imbalance between vasoconstrictive and vasodilative mediators with resulting endothelial dysfunction. These structural and functional abnormalities translate into increased systemic and pulmonary vascular resistance, and are now considered as key pathophysiological mechanism of HF, which have to date not been effectively therapeutically targeted.

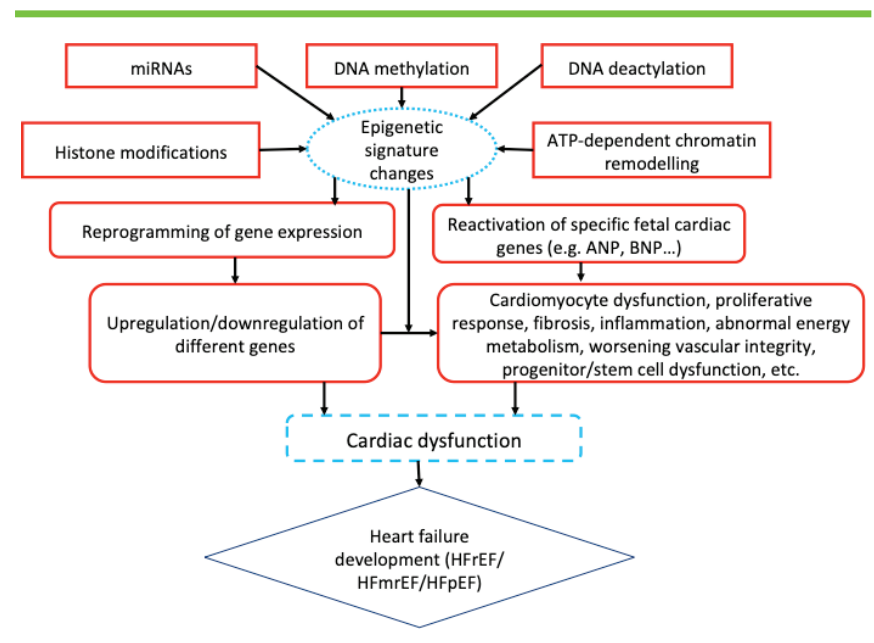

Figure 2. Epigenetic regulation in HF. Abbreviations: $\mathrm{a}-\mathrm{MHC}=$ the alpha-myosin heavy chain gene, SERCA2 = sarcoplasmic reticulum $\mathrm{Ca} 2+\mathrm{ATPase}$ gene

\section{Peripheral mechanisms as scientific targets}

Neurohormonal activation. Neurohormonal overactivity constitutes a fundamental pathophysiological mechanism involved in the origin and progression of HF. It remains the key therapeutic target, already underpinning multiple life-saving therapies for HFrEF worldwide.[10,27] Both the sympathetic nervous system (SNS) and the RAAS are activated reflexly during the onset of myocardial damage (due to reducing cardiac pumping shortly after the onset of myocardial infarction, myocarditis, etc.). These changes are thought to be adaptive during the initial stages and help to maintain acceptable cardiac output and tissue perfusion of critical vital organs such as the kidney and brain. Importantly, the features of neurohormonal activation are demonstrated at the systemic and peripheral level, namely molecular features of augmented aldosterone and adrenergic pathways have been demonstrated in the myocardium, but also in numerous peripheral tissues and organs, contributing to the progression of $\mathrm{HF}$ (e.g. vasculature, skeletal muscle, kidneys). However, a sustained chronic activation of RAAS and SNS translates into significant adverse consequences leading to progression of the HF syndrome, including peripheral vasoconstriction, sodium retention, haemodynamic alterations, skeletal dysfunction and renal dysfunction to name but a few.

Along with SNS and RAAS activation, there are adaptive and later maladaptive changes within the system of endogenous natriuretic peptides, which physiologically promote urinary sodium and water excretion, produce vasodilation, and inhibit pathological growth (e.g. hypertrophy and fibrosis).[28] In patients with HF, the response of overproduced natriuretic peptides is limited, therefore suggesting instead the therapeutic possibility of either administration of exogenous natriuretic peptide analogues or the inhibition of natriuretic peptide degradation (e.g. inhibition of neprilysin which degrades natriuretic peptides). The clinical effects of parenteral administration of exogenous natriuretic peptides (nesiritide, carperitide, ularitide) have not been conclusive, and further studies are ongoing, whereas the administration of the combination of an ARB (valsartan) with the neprilysin inhibitor sacubitril has been successful in improving symptoms and clinical outcomes in patients with HFrEF.[28] 


\section{Autonomic imbalance and abnormal reflex control. Along with neurohormonal activation, autonomic imbalance with abnormal reflex control constitutes another fundamental pathophysiological mechanism contributing critically to the origin and progression of HF. Autonomic imbalance is related to activation of the SNS and concurrent depression of parasympathetic nervous system. The features of autonomic imbalance are demonstrated at both the systemic and peripheral levels, namely molecular features of augmented adrenergic and depleted cholinergic signalling have been demonstrated in the myocardium, but also in numerous peripheral tissues and organs, contributing to the progression of HF (e.g. vasculature, skeletal muscle, kidneys, brain).}

Along with the progression of HF, we observe changes in the balance of reflexes controlling the function of the cardiovascular system, the pulmonary system and of skeletal muscles. Cardio-inhibitory reflexes, such as the baroreflex, are inhibited, whereas the sympatho-excitatory reflexes, such as central and peripheral chemoreceptor reflexes and ergoreceptor reflex, are augmented.[29,36,37] Again, these changes are to thought to be adaptive during the early stages of HF, but when they persist chronically they become maladaptive and further contribute to the unfavourable phenotype of HF, both within the myocardium and in the peripheral organs involved in the progression of HF.[30] Importantly, there is no consensus concerning the origin of autonomic imbalance and deranged reflex control in the course of HF, nor the parameters for effective modulation, such as dose, continuity of stimulation or blockade or selectivity as to which organs to target, which makes research in this field even more exciting, but also difficult.

The area of autonomic imbalance and deranged reflex control is currently under intensive investigation, both within the context of establishing reliable and accurate diagnostic methods which would allow us to translate sophisticated pathophysiology into simplified clinical implications, and also in the context of an identification of crucial targets for therapeutic interventions (both pharmacological agents and with implantable devices). Among the therapeutic approaches under investigations are the modulation of baroreflexes via carotid baroreceptor stimulation [32,33], carotid body ablation, renal sympathetic nerve ablation [34], vagal nerve stimulation [35], the activation of systemic cholinergic signalling (acetylocholinesterase inhibitors), and many more.

Inflammation. Immune activation is another prominent feature of $\mathrm{HF}$, with abnormalities seen primarily within the innate immune response (e.g. 'endotoxin hypothesis') and the imbalance between pro-inflammatory and anti-inflammatory mediators and reactions (favouring the later ones). Immune activation and inflammation are demonstrated in the systemic circulation, within the failing myocardium and peripheral tissues, both contributing to the progression of HF (e.g. skeletal muscles, vasculature, brain, kidneys). A pro-inflammatory state unfavourably affects myocardial function, exerts a negative inotropic effect, induces abnormalities in cardiac metabolism and energetics, induces cardiomyocyte hypertrophy, necrosis and apoptosis, and promotes myocardial remodelling.[38] Additionally, the activation of immune response in patients with HF promotes the endothelial dysfunction, autonomic imbalance, general body wasting, skeletal myopathy and anorexia, all increasing the risk of cachexia [39]. Until now clinical study results with interventions

\section{Table 1 Promising targets with preliminary clinical data in patients with heart failure.}

\begin{tabular}{l|l|l} 
Target & Therapeutic option & References
\end{tabular}

Promising targets with some clinical data

Hyperkalaemia induced by drugs

Patiromer, sodium zirconium 53,54

Sodium glucose cotransporter-2

(SGLT-2)

cGMP deficiency cyclosilicate

Inhibitors of SGLT-2 dapagliflozin

Pulmonary

hypertension

sGC stimulators (verciguat, riociguat)

Phosphodiesterase -5 inhibitor (tadalafil)

Endothelin agonist (macitentan)

Cellular/ mitochondrial ion homeostasis Increasing calcium sensitivity of myofilaments (omecamtiv mecarbil)

Contractility

Cardiac contractility modulation

Left atrial hypertension

Transcatheter interatrial shunt

Autonomic device

nervous system

Spinal cord stimulation

Future targets
Systemic

microvascular inflammation

Cardiometabolic functional abnormalities

\section{abnormalities}

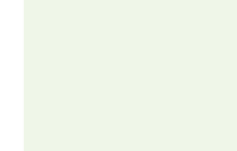

Cellular structura abnormalities (titin)

\section{Extracellular}

structural abnormalities (fibrosis, amyloid)

MicroRNA related with $\mathrm{HF}$ progression and myocardial remodelling

Genes related with Gene transfer through vector HF (e.g. SERCA2a, virus S100A1 and IPP-1) gamma inhibitors, IL-1beta mimicking agents (capadenoson, neladenoson) Carnitine palmitoyltransferase-1 inhibitors (etomoxir, perhexiline)

Fatty acid beta-oxidation inhibitor (trimetazidine)

Mitochondrial enhancer (elamipretide)

Genetic manipulation of an RNA 56 motif leading to up-regulation of compliant titins

Inhibition of TGF-beta-induced 56 fibrogenesis (pirfenidone)

Chemokine antagonists (anti-

Immuno-modulatory cytokines (interleukin (IL)-10, pentraxins, blockade with canakinumab or anakinra)

interfering with immune mechanisms have been inconclusive (e.g. anankira and canakikumab).[40-42]

Skeletal myopathy. According to 'muscle hypothesis', an acquired skeletal myopathy contributes to the symptomatology 


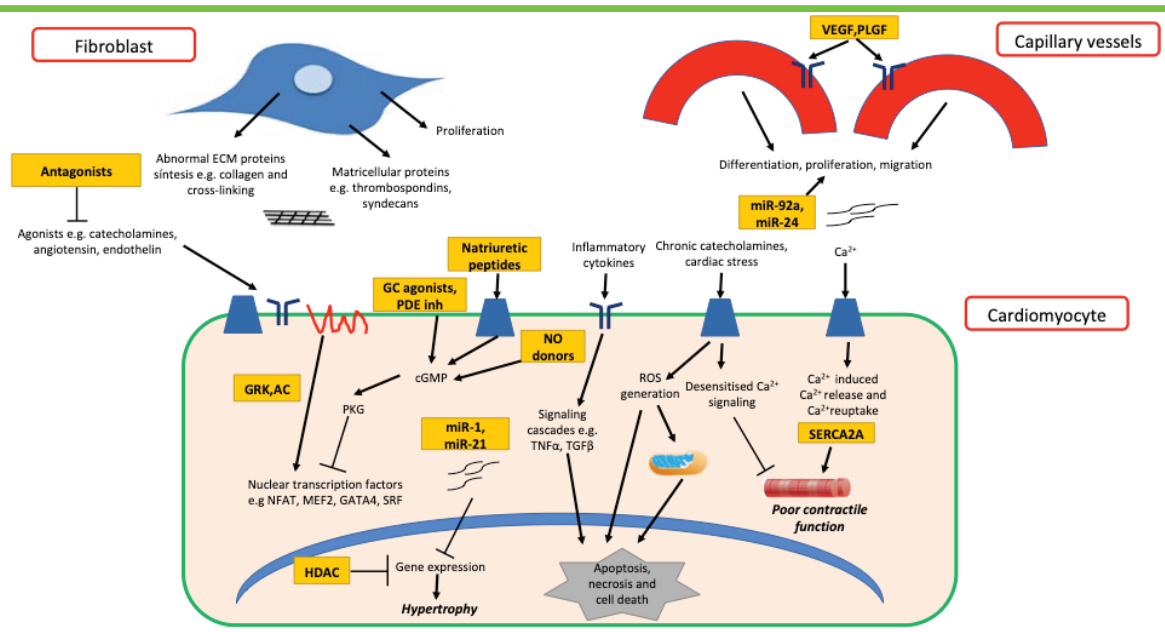

Figure 3. Pathophysiological mechanisms and key therapeutic targets (yellow boxes) in HF. The HF phenotype involves cardiomyocyte hypertrophy, abnormalities of excitation-contraction coupling, impaired cardiomyocyte viability, abnormal protein homeostasis, oxidative stress, energetic dysfunction, arrhythmia, extracellular matrix remodelling and chamber dyssynchrony. These changes are mediated by intracellular signalling pathways and the interactions of multiple cell types within the heart including fibroblasts, endothelial cells and their associated capillary network and extracellular matrix. Some of the key signaling pathways amenable to therapeutic targeting in HF are shown in the figure. VEGF (vascular endothelial growth factor), PLGF (placental growth factor), SERCA2A (sarcoplasmic/ endoplasmic reticulum Ca2+ ATPase), HDAC (histone deacetylase), GRK (G-protein coupled receptor kinase), AC (adenylate cyclase), NO (nitric oxide) and PDE inh (phosphodiesterase inhibitors).

and progression of HF. Skeletal myopathy is related to numerous changes within skeletal muscles, seen at different levels of complexity, including molecular (changed expression in structural elements of microfibrils and molecules involved in energetics and metabolic reactions); microscopic (structural and functional changes in sarcomeres, mitochondria and other organelles, abnormal regulation of the microcirculation, remodelling of extracellular matrix); and macroscopic with reduced muscle mass, increased fatigability, reflex-mediated exercise hyperventilation and impaired global exercise capacity. $[43,44]$ The origin of these abnormalities is not completely understood, but the following factors are presumed to contribute: neurohormonal activation, inflammation, autonomic imbalance with abnormal reflex control, including the augmented ergoreflex, a deranged microcirculation, catabolic-anabolic imbalance, abnormal energy metabolism and iron deficiency.

Improvement in the function of skeletal muscles constitutes a clinically relevant therapeutic target. Several attempts have been taken in order to least partially reverse the skeletal myopathy in $\mathrm{HF}$, some of them having a general influence on skeletal muscles (exercise programmes, including resistance exercise)[45], others more specifically targeting underlying pathomechanisms (e.g. intravenous iron supplementation, mitochondria enhancers, anabolic agents, metabolism modulators).

Co-morbidities. HF does not exist in isolation, but is commonly accompanied by numerous co-morbidities. Some of them may be just pathologies accompanying $\mathrm{HF}$ and occurring independently of the HF. However, some co-morbidities are related with the progression of $\mathrm{HF}$, where intrinsic elements of HF pathology predispose to the development of other diseases (e.g. iron deficiency, insulin resistance and overt diabetes, hyponatraemia), or treatment applied in patients with HF leads to other abnormalities (e.g. hyperkalaemia).
Anaemia constitutes a frequent co-morbidity, its prevalence is related with the progression of $\mathrm{HF}$ and is associated with increased morbidity and mortality.[46] Although the origin of anaemia in HF is not comprehensively established, neurohormonal activation, pro-inflammatory mediators and renal dysfunction are presumed to contribute to its development.[47] Anaemia remains a clinically important target, despite erythropoiesis-stimulating agents having failed to show an adequate safety and efficacy in HF. There are some other agents being investigated with some promising results, including intravenous iron supplementation in case of iron deficiency anaemia.

Iron deficiency (ID) should be perceived as a separate common and ominous co-morbidity in patients with HF. ID, regardless of concomitant anaemia, accelerates the progression of $\mathrm{HF}$, significantly contributes to skeletal myopathy and predisposes to unfavourable clinical outcomes.[48,49] In spite of intravenous iron supplementation already studied in some groups of patients with HF [50], the other molecules interfering with metabolic pathways orchestrated by iron are of special scientific and clinical interest (see also chapter 19). Among electrolyte abnormalities occurring in the course of HF, hyponatraemia and hyperkalaemia (see also chapter 17) are of a particular clinical relevance, and hence several therapeutic approaches have been designed and investigated.[51,52]

\section{Conclusions}

The pathophysiology of HF is currently being intensively investigated, with the identification of new relevant mechanisms, some of them emerging as potential therapeutic targets. The most recent developments are briefly summarized in Table 1 and Figure 3.

\section{Declarations of interest}

The authors declare no conflict of interest. 


\section{Acknowledgements}

The authors state that they abide by the authors' responsibilities and ethical publishing guidelines of the International Cardiovascular Forum Journal.[61]

\section{References}

1. McMurray J, Petrie M, Swedberg, Komajda M, Anker S, Gardner R. Heart Failure. Chapter 23. THE ESC TEXTBOOK OF CARDIOVASCULAR MEDICINE 2nd edition. 2009. Oxford University Press.

2. Caro CG. The Mechanics of the Circulation. 2nd ed. New York: Cambridge University Press; 2011.

3. Cohn JN, Ferrari R, Sharpe N. Cardiac remodelling - concepts and clinical implications: a consensus paper from an international forum on cardiac remodeling. Behalf of an International Forum on Cardiac Remodeling. J Am Coll Cardiol 2000; 35: 569-82.

4. Verdecchia P, Angeli F, Cavallini C, Gattobigio R, Gentile G, Staessen JA, et al. Blood pressure reduction and renin-angiotensin system inhibition for prevention of congestive heart failure: a meta-analysis. Eur Heart $\mathrm{J}$ 2009;30:679-88

5. Ponikowski P, Voors AA, Anker SD, Bueno H, Cleland JGF, Coats AJS, et al. 2016 ESC Guidelines for the diagnosis and treatment of acute and chronic heart failure: The Task Force for the diagnosis and treatment of acute and chronic heart failure of the European Society of Cardiology (ESC) Developed with the special contribution of the Heart Failure Association (HFA) of the ESC. Eur Heart J 2016;37(27):2129-200.

6. Stone GW, Lindenfeld J, Abraham WT, et al. Transcatheter MitralValve Repair in Patients with Heart Failure. N Engl J Med. 2018;379(24): 2307-2318.

7. Breithardt OA, Stellbrink C, Franke A, Balta O, Diem BH, Bakker P, et al. Acute effects of cardiac resynchronization therapy on left ventricular Doppler indices in patients with congestive heart failure. Am Heart $\mathrm{J}$ 2002;143:34-44.

8. Auricchio A, Stellbrink C, Sack S, Block M, Vogt J, Bakker P, et al. Longterm clinical effect of hemodynamically optimized cardiac resynchronization therapy in patients with heart failure and ventricular conduction delay. J Am Coll Cardiol 2002;39:2026-33.

9. Tamargo J, López-Sendón J. Novel therapeutic targets for the treatment of heart failure. Nat Rev Drug Discov 10(7):536-555, 2011

10. Tamargo J, Caballero R, Delpón E. New drugs in preclinical and early stage clinical development in the treatment of heart failure, Expert Opinion on Investigational Drugs 2019; 28:1, 51-71

11. Maack $C$, Eschenhagen $\mathrm{T}$, Hamdani $\mathrm{N}$, et al. Treatments targeting inotropy. Eur Heart J. 2018 Oct 8. doi: 10.1093/eurheartj/ehy600. [Epub ahead of print]

12. Nishida K, Yamaguchi Otsu K, et al. Crosstalk between autophagy and apoptosis in heart disease. Circ Res 2008; 103: 343-51

13. Segura AM, Frazier OH, Buja LM. Fibrosis and heart failure. Heart Fail Rev. $2014 ; 19(2): 173-85$ '.

14. Levick SP, Brower GL. Regulation of matrix metalloproteinases is at the heart of myocardial remodeling. Am J Physiol Heart Circ Physiol 2008; 295: H1375-H1376

15. Spinale FG, Villarreal F. Targeting matrix metalloproteinases in heart disease: lessons from endogenous inhibitors. Biochem Pharmacol. 2014;90(1):7-15.

16. Smith JG. Molecular Epidemiology of Heart Failure: Translational Challenges and Opportunities. JACC Basic TransI Sci. 2017;2(6):757-769

17. Zhou SS, Jin JP, Wang JQ, et al. miRNAS in cardiovascular diseases: potential biomarkers, therapeutic targets and challenges. Acta Pharmacol Sin. 2018;39(7):1073-1084.

18. Napoli C., Grimaldi V., De Pascale M.R., Sommese L., Infante T., Soricelli A. Novel epigenetic-based therapies useful in cardiovascular medicine. World J. Cardiol. 2016;8(2):211-219.

19. Di Salvo T.G., Haldar S.M. Epigenetic mechanisms in heart failure pathogenesis. Circ. Heart Fail. 2014;7(5):850-863.

20. Berezin A. Epigenetics in heart failure phenotypes. BBA Clin. 2016;6:31-7.

21. Bayliss J, Norell M, Canepa-Anson R, Sutton G, Poole-Wilson P. Untreated heart failure: clinical and neuroendocrine effects of introducing diuretics. $\mathrm{Br}$ Heart J 1987;57:17-22.

22. Senni M, Paulus WJ, Gavazzi A, et al. New strategies for heart failure with preserved ejection fraction: the importance of targeted therapies for heart failure phenotypes. Eur Heart J 2014;35:2797-815.

23. Pries AR, Reglin B. Coronary microcirculatory pathophysiology: can we afford it to remain a black box? Eur Heart J 2017;38:478-88.

24. Hogg K, McMurray J. Neurohumoral pathways in heart failure with preserved systolic function. Prog Cardiovasc Dis 2005;47:357-66.

25. Bavishi C, Chatterjee S, Ather S, Patel D, Messerli FH. Beta-blockers in heart failure with preserved ejection fraction: a meta-analysis. Heart Fail Rev 2015;20:193-201.

26. Redfield MM, Jacobsen SJ, Borlaug BA, Rodeheffer RJ, Kass DA. Age- and gender related ventricular-vascular stiffening: a community-based study. Circulation 2005; 112:2254-2262.
27. Yancy CW, Jessup M, Bozkurt B, et al. 2017 ACC/AHA/HFSA Focused Update of the 2013 ACCF/AHA Guideline for the Management of Heart Failure: A Report of the American College of Cardiology/American Heart Association Task Force on Clinical Practice Guidelines and the Heart Failure Society of America. J Am Coll Cardiol. 2017;70(6):776-803.

28. McMurray JJ. Neprilysin inhibition to treat heart failure: a tale of science, serendipity, and second chances. Eur J Heart Fail 2015;17:242-7.

29. Watson AM, Hood SG, May CN. Mechanisms of sympathetic activation in heart failure. Clin Exp Pharmacol Physiol 2006;33:1269-74

30. Esler M. The 2009 Carl Ludwig Lecture: Pathophysiology of the human sympathetic nervous system in cardiovascular diseases: the transition from mechanisms to medical management. J Appl Physiol 2010;108:227-37.

31. J.N. Cohn, M.A. Pfeffer, J. Rouleau, et al. Adverse mortality effect of central sympathetic inhibition with sustained-release moxonidine in patients with heart failure (MOXCON). Eur J Heart Fail 2003; 5: 659-667.

32. Grassi G, Brambilla G, Pizzalla DP, Seravalle G. Baroreflex Activation Therapy in Congestive Heart Failure: Novel Findings and Future Insights. Curr Hypertens Rep. 2016;18(8):60.

33. Singh JP, Kandala J, Camm AJ. Non-pharmacological modulation of the autonomic tone to treat heart failure. Eur Heart J. 2014 Jan;35(2):77-85.

34. Böhm M, Ewen S, Mahfoud F. Renal Denervation for Chronic Heart Failure: Background and Pathophysiological Rationale. Korean Circ J. 2016;47(1):9-15.

35. Premchand RK, Sharma K, Mittal S. Extended Follow-Up of Patients With Heart Failure Receiving Autonomic Regulation Therapy in the ANTHEM-HF Study. J Card Fail. 2016 ;22(8):639-42.

36. Paula-Ribeiro $M$, Rocha $A$. The peripheral-central chemoreflex interaction: where do we stand and what is the next step?. J Physiol. 2016;594(6):1527-8.

37. Del Rio R, Andrade DC, Toledo C, et al. Carotid Body-Mediated Chemoreflex Drive in The Setting of low and High Output Heart Failure. Sci Rep. 2017;7(1):8035.

38. Sattler S, Fairchild P, Watt FM, Rosenthal N, Harding SE. The adaptive immune response to cardiac injury-the true roadblock to effective regenerative therapies? NPJ Regen Med 2017;2:19.

39. Jankowska EA, Ponikowski P, Piepoli MF, Banasiak W, Anker SD, PooleWilson PA. Autonomic imbalance and immune activation in chronic heart failure - pathophysiological links. Cardiovasc Res. 2006;70(3):434-45.

40. Ridker PM, Everett BM, Thuren T, et al. Antiinflammatory therapy with canakinumab for atherosclerotic disease. N Engl J Med 2017;377:11191131.

41. Van Tassell BW, Abouzaki NA, Oddi Erdle C, et al. Interleukin-1 blockade in acute decompensated heart failure. J Cardiovasc Pharmacol 2016;67:544-551.

42. Abbate A, Kontos MC Grizzard JD, et al Interleukin-1 blockade with anakinra to prevent adverse cardiac remodeling after acute myocardial infarction (Virginia Commonwealth University Anakinra Remodeling Trial [VCU-ART] Pilot Study). Am J Cardiol 2010;105:1371-1377.

43. Josiak K, Jankowska EA, Piepoli MF, Banasiak W, Ponikowski P. Skeletal myopathy in patients with chronic heart failure: significance of anabolicandrogenic hormones. J Cachexia Sarcopenia Muscle. 2014;5(4):287-96.

44. Ponikowski PP, Chua TP, Francis DP, Capucci A, Coats AJ, Piepoli MF. Muscle ergoreceptor overactivity reflects deterioration in clinical status and cardiorespiratory reflex control in chronic heart failure. Circulation. 2001;104(19):2324-30.

45. von Haehling S, Steinbeck L, Doehner W, et al. Muscle wasting in heart failure: an overview. Int J Biochem Cell Biol. 2013;45:2257-65.

46. Groenveld HF, Januzzi JL, Damman K, et al. Anemia and mortality in heart failure patients a systematic review and meta-analysis. J Am Coll Cardiol 2008;52:818-27.

47. Anand IS, Gupta P. Anemia and Iron Deficiency in Heart Failure: Current Concepts and Emerging Therapies. Circulation. 2018;138(1):80-98.

48. Jankowska EA, Malyszko J, Ardehali $\mathrm{H}$, et al. Iron status in patients with chronic heart failure. Eur Heart J 2013;34:827-34.

49. Jankowska EA, von Haehling S, Anker SD, Macdougall IC, Ponikowski $P$. Iron deficiency and heart failure: diagnostic dilemmas and therapeutic perspectives. Eur Heart J 2013;34:816-29.

50. Jankowska EA, Tkaczyszyn M, Suchocki T, et al. Effects of intravenous iron therapy in iron-deficient patients with systolic heart failure: a meta-analysis of randomized controlled trials. Eur J Heart Fail. 2016 Jul;18(7):786-95.

51. Urso C, Brucculeri S, Caimi G. Acid-base and electrolyte abnormalities in heart failure: pathophysiology and implications. Heart Fail Rev. 2015;20(4):493-503.

52. Grodin JL. Pharmacologic Approaches to Electrolyte Abnormalities in Heart Failure. Curr Heart Fail Rep. 2016;13(4):181-9.

53. Bakris G, Pitt B, Weir M, et al. Effect of patiromer on serum potassium level in patients with hyperkalemia and diabetic kidney disease: the amethystDN randomized clinical trial. JAMA. 2015;314:151-61.

54. Sr A, Singh B, Lavin P, Stavros F, Rasmussen H. A phase 2 study on the treatment of hyperkalemia in patients with chronic kidney disease suggests that the selective potassium trap, Zs-9, is safe and efficient. Kidney Int. 2015;88:404-11. 
55. Kosiborod M, Cavender MA, Fu AZ, et al. Lower Risk of Heart Failure and Death in Patients Initiated on Sodium-Glucose Cotransporter-2 Inhibitors Versus Other Glucose-Lowering Drugs: The CVD-REAL Study (Comparative Effectiveness of Cardiovascular Outcomes in New Users of SodiumGlucose Cotransporter-2 Inhibitors). Circulation. 2017 Jul 18;136(3):249259.

56. Lam CSP, Voors AA, de Boer RA, Solomon SD, van Veldhuisen DJ. Heart failure with preserved ejection fraction: from mechanisms to therapies. Eur Heart J. 2018;39(30):2780-2792.

57. Teerlink JR, Felker GM, McMurray JJV, et al. Acute Treatment With Omecamtiv Mecarbil to Increase Contractility in Acute Heart Failure: The ATOMIC-AHF Study. J Am Coll Cardiol. 2016;67(12):1444-1455.

58. Tschöpe C, Kherad B, Klein O, et al. Cardiac contractility modulation: mechanisms of action in heart failure with reduced ejection fraction and beyond. Eur J Heart Fail. 2019;21(1):14-22.

59. Shah SJ, Feldman T, Ricciardi MJ, et al. One-Year Safety and Clinical Outcomes of a Transcatheter Interatrial Shunt Device for the Treatment of Heart Failure With Preserved Ejection Fraction in the Reduce Elevated Left Atrial Pressure in Patients With Heart Failure (REDUCE LAP-HF I) Trial: A Randomized Clinical Trial. JAMA Cardiol. 2018;3(10):968-977.

60. Rosik J, Szostak B, Machaj F, Pawlik A. Potential targets of gene therapy in the treatment of heart failure. Expert Opin Ther Targets. 2018;22(9):811-816.

61. Shewan LG, Coats AJS, Henein MY. Authors' Responsibilities and Ethical Publishing. International Cardiovascular Forum Journal 2018;13:3-4, DOI: $10.17987 /$ icfj.v13i0.525 